\title{
Imaging-guided Percutaneous Renal Mass Biopsy】 predictive factors of diagnosis, accuracy , safety, and changes of the treatment
}

Liefu Ye ( $\nabla$ yeliefu@fjmu.edu.cn )

Fujian Provincial Hospital

\section{Songmao Chen}

Provincial Clinical Medical College of Fujian Medical University

\section{Yuandong Chen}

Xiamen Maternity and Child Health Care Hospital

Qingguo Zu

Fujian Provincial Hospital

\section{Zesong Yang}

Fujian Provincial Hospital South Branch

\section{Minxiong Hu}

Fujian Provincial Hospital

\section{Research Article}

Keywords: Renal cell carcinoma, Renal tumor biopsies, treatment

Posted Date: March 2nd, 2022

DOI: https://doi.org/10.21203/rs.3.rs-1391539/v1

License: (1) This work is licensed under a Creative Commons Attribution 4.0 International License.

Read Full License 


\section{Abstract}

Objective $\triangle$ To evaluate the predictive factors of imaging-guided percutaneous renal mass biopsy(PRMB) for diagnosis and its accuracy, safety $\mathbb{T}$ To analysis changes in the treatment of renal tumors with biopsy.

Method $\backslash$ We retrospectively collected clinical data of patients with PRMB. The diagnosis rate of biopsy, pathological results, and complications were analyzed. Suspicious factors affecting the diagnosis of the biopsy were studied by logistic regression analysis. Including the age, gender, tumor characteristics, length of the biopsy specimen, and the number of biopsy cores between the diagnostic group and the non-diagnostic group. The surgical pathology reports were used as the standard to compare the accuracy of the biopsy. Analyze the changes in the treatment plan with PRMB.

Results $\triangle$ A total of 109 patients were collected. Multivariate Logistic regression analysis showed that the number of biopsy cores $(p=0.005)$ and the tumor solid feature $(p=0.038)$ were the independent predictors of the diagnostic biopsy. 16 patients of the diagnostic group underwent surgical treatment and obtained postoperative pathology. Compared with the surgical pathology, The accuracy of biopsy in identifying malignancies, histotyping, and grading were $100 \%(16 / 16) \llbracket 85.7 \% \bigotimes 12 / 14 \rrbracket$ and $50 \% \varangle 2 / 4 \rrbracket$ respectively. 10 cases $₫ 9.2 \% \bigotimes$ changed the treatment plan because of biopsy. There were 10 cases $₫ 9.2 \% \varangle$ with complications.

Conclusions $₫$ The number of biopsy cores and the solid feature of tumor were the independent predictors of diagnostic biopsy. PRMB has high diagnostic accuracy in identifying malignancies, and histotyping. The PRMB can change part of the treatment plans of patients with renal tumors. The complication rate is low and hematoma is the most common complication.

\section{Introduction}

The global incidence of renal tumors is increasing year by year. At present, the annual incidence of renal malignant tumors in Western countries is increasing at a rate of $2 \%$ per year ${ }^{[1]}$. Approximately 7,380 newly diagnosed cases and 14,770 deaths were added in the U.S. in $2019^{[2]}$. The current diagnosis of renal tumor mainly relies on imaging examinations such as ultrasonography (US), abdominal computerized tomography (CT), and abdominal magnetic resonance imaging (MRI). However, imaging examination has limitations for rare kidney tumors, and pathological diagnosis cannot be obtained. With the application of needle biopsy technology mature, more and more clinicians apply percutaneous renal mass biopsy (PRMB) for some specific cases (small renal masses, patients who cant undergo surgery and will be treated minimal invasive procedures, those with suspected metastases and others). This study retrospectively collected the clinical information of patients who underwent PRMB under the guidance of imaging in our hospital, statistically calculated the diagnosis rate of PRMB, the pathological reports of PRMB, and complications. The predictive factors of the diagnostic biopsy were further analyzed, as well as the changing of treatment plan because of PRMB. 


\section{Objects And Methods \\ 1.1 Patients selection}

This study retrospectively collected the clinical data of patients who underwent PRMB at Fujian Provincial Hospital from January 2012 to July 2020.

Exclusion factor $\bigotimes$ Patients undergoing previous surgical treatment for renal cancer and were found tumor recurrence.

\subsection{Clinical data selection}

Clinic information includes the patient's gender, age, renal tumor diameter, side (left renal/right renal), Exophytic appearance (yes/no), number of biopsy cores, Tumor features (solid/cystic- solid), and length of puncture specimen. Pathological information including diagnosis of malignant or benign, histological type, histological grade were also collected. According to symptom and imaging examination of patients after biopsy, we recorded complications such as perirenal hematoma, subrenal hematoma, hematuria, backache, pneumothorax, needle tract seeding, and so on.

\subsection{PRMB process}

Lateral decubitus position was adopted according to the side of the patient's renal tumor, and the needle position and direction were guided by the US or CT. the $18 \mathrm{G}$ lateral cutting through-type autobiopsy gun (BARD Company, USA) was used for biopsy. After the biopsy, the patient is transferred to the ward for monitoring of vital signs, dynamic review of blood routine, and coagulation markers. It is needed to stay in bed for 1-2 days and take symptomatic treatment for hemostasis and pain.

\subsection{Grouping standard}

(1)the diagnostic group $\mathbb{T}$ The biopsy pathological results can distinguish tumors from benign or malignant; the non-diagnostic group $\mathbb{T}$ The pathological biopsy results are useless to differentiate benign or malignant renal tumors.

(2)Treatment changed group: The treatment plan was changed due to the pathological results of the biopsy. It is referred to the addition or deletion of any of the following items in the preoperative treatment plan: surgery, radiotherapy, and chemotherapy, targeted therapy, ablation therapy, as well as changes of the surgical plan, targeted therapy plan, or radiotherapy and chemotherapy plan; Treatment unchanged group: The biopsy pathological results did not change the treatment plan formulated before the operation. 


\section{5 statistical method}

SPSS ver.21.0 (IBM Corp, Armonk, NY) was used for all statistical analyses. Continuous variables were reported as mean $\pm S D$ and/or median(interquartile range区IQR) and analyzed with Mann-Whitney $U$ or Kruskal-Wallis tests as applicable. Univariate and multivariate logistic regression models were used to determine the independent association of variables with the presence of diagnostic pathologic findings. All statistical tests were two-tailed, with $p<0.05$ being considered to be significant. concordance rate with the surgical excision diagnosis was calculated.

\section{Results}

\subsection{Clinical data.}

A total of 109 patients were included, 79 patients were male, and 30 patients were female. The median age was 59.6 years (range: 2-86 years). 102 patients completed biopsy under ultrasound guidance, and 7 patients completed biopsy under CT guidance. Among the results of 109 cases, 95 cases were in the diagnostic group and 14 cases were in the non-diagnostic group. The diagnostic rate was $87.2 \%$ ( Table 1).

Table 1

Baseline patient and lesion characteristics 


\begin{tabular}{|c|c|}
\hline variables & overall $(n .=109$ \\
\hline \multicolumn{2}{|l|}{ Gender } \\
\hline Male & $79 \otimes 72.5 \% \rrbracket$ \\
\hline Female & $30 \otimes 27.5 \% \rrbracket$ \\
\hline Age『years (IQR) & $60 \rrbracket 50-72 \rrbracket$ \\
\hline Tuomor diameter,cm (IQR) & $4.4 \llbracket 2.4-8 \rrbracket$ \\
\hline \multicolumn{2}{|l|}{ Lesion location } \\
\hline Left renal & $53 \rrbracket 48.6 \% \rrbracket$ \\
\hline Right renal & $56 \rrbracket 51.4 \% \rrbracket$ \\
\hline \multicolumn{2}{|l|}{ Tuomor features } \\
\hline Solid & $85 \bigotimes 78.0 \% \rrbracket$ \\
\hline Cystic-Solid & $24 \rrbracket 22.0 \% \rrbracket$ \\
\hline \multicolumn{2}{|l|}{ Exophytic appearance, (vs no) } \\
\hline Yes & $81 \otimes 74.30 \% \bigotimes$ \\
\hline No & $28 \rrbracket 25.7 \% \rrbracket$ \\
\hline \multicolumn{2}{|l|}{ Type of imaging used } \\
\hline CT & $7 \rrbracket 6.4 \% \rrbracket$ \\
\hline US & 102ه93.6\%区 \\
\hline \multicolumn{2}{|l|}{ N. of biopsy cores } \\
\hline 1 & $10 \rrbracket 9.2 \% \rrbracket$ \\
\hline 2 & $28 \rrbracket 25.7 \% \rrbracket$ \\
\hline 3 & $48 ه 44.0 \% \rrbracket$ \\
\hline 4 & $17 \nabla 15.6 \% \bigotimes$ \\
\hline 5 & $5 \rrbracket 4.6 \% \rrbracket$ \\
\hline 6 & $1 \rrbracket 0.9 \% \rrbracket$ \\
\hline Specimen length,mm (IQR) & $15 \rrbracket 15-20 \rrbracket$ \\
\hline \multicolumn{2}{|l|}{ Treatment } \\
\hline Surgery & $16 \bigotimes 14.7 \% \rrbracket$ \\
\hline Radiofrequency ablation & $48 \rrbracket 44.0 \% \rrbracket$ \\
\hline
\end{tabular}


Data presented as median (IQR) or as number of cases (prevalence).

$\mathrm{IQR}=$ interquartile range.

\subsection{Pathological results of the biopsy in the diagnostic group}

The pathological results of the diagnostic group are shown in Table 2. 85 cases were diagnosed as malignant tumors, in which 14 cases of renal cell carcinoma failed to further clarify the histologic type. 43 cases were diagnosed as renal clear cell carcinoma or papillary renal cell carcinoma. The histological grade of $41(95.3 \%)$ cases was confirmed. Two cases could not be graded due to less tissue. There were 10 cases $(9.2 \%)$ with benign lesions. Only 16 patients in the diagnostic group underwent surgery and obtained postoperative pathological reports. With postoperative pathology as the gold standard, The accuracy of biopsy in identifying malignancies, histotyping, and grading were 100\% (16/16), 85.7\% $(12 / 14)$, and $50 \%(2 / 4)$.

Table 2

Pathology of biopsy in the diagnostic group 


\begin{tabular}{|c|c|c|c|}
\hline Histological types $₫ n=95 \rrbracket$ & $\mathrm{n},(\%)$ & Fuhrman grade $₫ n=43 \rrbracket$ & $\mathrm{n},(\%)$ \\
\hline Renal cell tumor & & I & $28(65.1)$ \\
\hline Clear cell renal cell carcinoma & $39(41.1)$ & II & $10(23.2)$ \\
\hline Papillary renal cell carcinoma & $4(4.2)$ & III & $2(4.7)$ \\
\hline Chromophobe renal cell carcinoma & $4(4.2)$ & IV & $1(2.3)$ \\
\hline Collecting duct carcinoma & $2(2.1)$ & Unable to grade & $2(4.7)$ \\
\hline MiT family translocation renal cell carcinoma & $1(1.1)$ & & \\
\hline Renal cell carcinoma $\quad \nabla$ & $14(14.7)$ & & \\
\hline Oncocytoma & $2(2.1)$ & & \\
\hline \multicolumn{4}{|l|}{ Nephroblastic tumors } \\
\hline Nephroblastoma & $1(1.1)$ & & \\
\hline \multicolumn{4}{|l|}{ Mesenchymal tumors } \\
\hline Angiomyolipoma & $5(5.3)$ & & \\
\hline Epithelioid angiomyolipoma & $1(1.1)$ & & \\
\hline Perivascular epithelioid cell tum $\bowtie$ Pecoma) & $1(1.1)$ & & \\
\hline Myeloid sarcoma & $1(1.1)$ & & \\
\hline Leiomyosarcoma & $1(1.1)$ & & \\
\hline \multicolumn{4}{|l|}{ Others } \\
\hline Compensatory renal hypertrophy & $1(1.1)$ & & \\
\hline Specific inflammatory granuloma & $2(2.1)$ & & \\
\hline Diffuse large B-cell lymphoma & $3(3.2)$ & & \\
\hline Squamous Cell Carcinoma of Renal Pelvis & $1(1.1)$ & & \\
\hline Urothelial carcinoma of renal pelvis & $1(2.1)$ & & \\
\hline Metastatic tumors & $3(3.2)$ & & \\
\hline Other malignant tumors of the kidney & $8(8.4)$ & & \\
\hline
\end{tabular}


A total of 14 cases failed to obtain the pathological diagnosis and the reasons were as follows: 5 cases (35.7\%) were punctured to normal renal tissue, 4 cases $(28.6 \%)$ were not obtained enough tissue for diagnosis, 2 cases $(14.2 \%)$ were obtained necrotic bleeding tissue, 2 cases $(14.3 \%)$ were obtained mesenchymal tissue, and 1 case $(7.1 \%)$ were obtained inflammatory infiltration tissue.

\subsection{Factors associated with the diagnostic biopsy of PRMB}

Table 3 shows the univariate and multivariate analyses for the diagnostic biopsy results at the PRMB. The univariate analysis showed that the exophytic appearance $(P=0.041)$ and the number of biopsy cores $(P=0.001)$ were significantly associated with the diagnostic biopsy. The exophytic appearance, the number of biopsy cores, and Tumor features with $\mathrm{P}<0.1$ of univariate analysis were included in multivariate Logistic regression analysis. The multivariate analysis results showed that the number of biopsy cores (OR: $9.22 ; 95 \% \mathrm{Cl}, 1.92-44.15, \mathrm{p}=0.005$ ) and tumor solid feature (OR: $4.68 ; 95 \% \mathrm{Cl}, 1.22-$ $17.99, p=0.038$ ) were independent predictive factors of the diagnostic biopsy.

\section{Table 3}

Univariable and multivariable analysis of factors associated with a diagnostic biopsy at the time of the initial renal tumor biopsy.

\begin{tabular}{|c|c|c|c|c|c|c|}
\hline & \multicolumn{3}{|c|}{ Univariate analysis } & \multicolumn{3}{|c|}{ Multivariate analysis } \\
\hline Patients characteristics & OR & $95 \% \mathrm{Cl}$ & $\mathrm{p}$ & OR & $95 \% \mathrm{Cl}$ & $\mathrm{p}$ \\
\hline Gender & 1.46 & $0.38-5.63$ & 0.59 & & & \\
\hline Age, yr (per 1-yr increase) & 0.98 & 0.94-1.02 & 0.34 & & & \\
\hline Diameter , cm \per 1-cm increase $\rrbracket$ & 1.14 & 0.94-1.39 & 0.18 & & & \\
\hline Side $\rrbracket$ (right vs left) $\rrbracket$ & 1.48 & $0.48-4.60$ & 0.45 & & & \\
\hline Tumor features, (solid vs Cystic- solid) & 3.21 & $\begin{array}{l}0.99- \\
10.40\end{array}$ & 0.05 & 4.68 & $\begin{array}{l}1.22- \\
17.99\end{array}$ & 0.025 \\
\hline Exophytic appearance, (yes vs no) & 3.32 & $\begin{array}{l}1.05- \\
10.49\end{array}$ & 0.04 & 2.79 & $\begin{array}{l}0.74- \\
10.43\end{array}$ & 0.128 \\
\hline 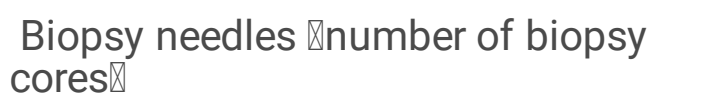 & 10.00 & $\begin{array}{l}2.43- \\
41.22\end{array}$ & 0.001 & 9.22 & $\begin{array}{l}1.92- \\
44.15\end{array}$ & 0.005 \\
\hline $\begin{array}{l}\text { Specimen length, cm『per 1-cm } \\
\text { increase } \mathbb{}\end{array}$ & 1.12 & $0.96-1.30$ & 0.14 & & & \\
\hline
\end{tabular}




\subsection{Changes of the treatment plan after biopsy}

Because the pathological results after the biopsy were not consistent with the previous imaging diagnosis, 10 cases changed treatment plans as shown in Table 4 for details.

Table 4

Changes of treatment plan on account of PRMB 
Renal cancer with lung and brain metastasis.(Imaging showed a single renal tumor and multiple nodules in the lung and brain.)
The biopsy showed renal angiomyolipoma, combined with the results of cerebrospinal fluid examination and other related tuberculosis examinations, considering that the lung and brain nodules were infected with tuberculosis, and anti-tuberculosis treatment
Lymphomaßpostoperation of nasopharyngeal carcinoma surgery, PET-CT showed increased metabolism in both kidneys and spleen $\rrbracket$
The biopsy showed that the kidney lesion was a metastatic tumor of nasopharyngeal carcinoma
Recurrence of breast cancer (postoperation of breast cancer surgery, imaging revealed multiple organ metastases in the armpit, neck, lung, liver, and kidney)
The biopsy showed a primary renal malignant tumor
Malignant tumor of renal (The imaging results consider the possibility of malignant tumors in the kidney)
Biopsy results show benign lesions, avoid surgical treatment
Renal cell carcinoma (The imaging results consider the possibility of renal cell carcinoma)
The biopsy showed renal pelvic cancer. One case of renal pelvic squamous cell carcinoma changed the surgical treatment plan, and the other case of urothelial carcinoma changed from targeted therapy to chemotherapy
Lymphoma (The imaging results consider the renal mass as a lymphoma)
The biopsy result was myeloid sarcoma, and the chemotherapy regimen was changed 
Complications occurred in 10 cases(9.2\%). Among them, 4 cases with perirenal hematoma and 1 case with subcapsular hematoma were self-absorbed and did not need further treatment. Postoperative transient fever occurred in 1 case and gross hematuria occurred in 1 case, however, the symptoms of these 2 cases disappeared spontaneously without treatment. 1 case developed urinary tract infection symptoms such as frequent urination and urgency after surgery, which was cured after oral antibiotic treatment. 2 cases had obvious lumbago after the operation, and the symptoms were relieved after analgesic treatment. (Table 5)

The severity of complications was based on the Clavien-Dindo complication grading system. 9 cases were Clavien-Dindo grade 1, and 1 case was Clavien-Dindo grade 2 . There were no serious complications (Clavien-Dindo grade > 2), and no needle tract seeding had been found after a median follow-up time of 46 months (range: 10-76months).

Table 5

Adverse events reported after renal tumor biopsy

\begin{tabular}{|c|c|c|c|}
\hline Type $\ n=10 \rrbracket$ & $\mathrm{n} \otimes \mathbb{Q} \% \square$ & Complication classification $\rrbracket n=10 \bigotimes$ & $\mathrm{n} \otimes \square \% \bigotimes$ \\
\hline Perirenal hematoma & $4 \rrbracket 40.0 \rrbracket$ & Clavien-Dindo grade I & $9 \rrbracket 90.0 \rrbracket$ \\
\hline
\end{tabular}

\begin{tabular}{|c|c|c|c|}
\hline Subcapsular hematoma & $1 \otimes 10.0 \otimes$ & Clavien-Dindo grade II & $1 \otimes 10.0 \otimes$ \\
\hline Fever & $1 \otimes 10.0 \rrbracket$ & Clavien-Dindo grade III & $0 \otimes 0 \otimes$ \\
\hline Self-limited hematuria & $1 \otimes 10.0 \otimes$ & Clavien-Dindo grade IV & $0 \otimes 0 \otimes$ \\
\hline Urinary tract infections & $1 \otimes 10.0 \otimes$ & & \\
\hline Significant low back pain & $2 \rrbracket 20.0 \rrbracket$ & & \\
\hline
\end{tabular}

\section{Discuss}

Currently, an increase in the detection rate of renal tumors was observed over the years. Previous views believed that the majority of renal tumors are malignant and the treatment was relatively radical. However, as more and more renal tumors are detected, many studies have found that benign ones are not rare $(20 \%-30 \%)^{[3-5]}$. Although most malignant renal tumors can be identified by advanced imaging examinations (such as $\mathrm{CT}, \mathrm{MRI}$, etc.), there are still many renal malignancies that cannot be differentiated because of secondary changes such as internal hemorrhage, necrosis, and cystic changes are infrequent in these tumors. Additionally, Part of benign renal tumors has similar imaging findings with renal cell carcinoma, such as fat-deficient renal hamartoma, metanephric adenoma, and eosinophilic adenoma. Bauman et al. reported that approximately $14.1 \%$ (129 cases/916 cases) of renal benign tumors confirmed by postoperative pathology were misdiagnosed as malignant tumors by imaging ${ }^{[6]}$. PRMB can 
not only make up for the deficiency of imaging but also help clinicians to obtain pathological information in advance to guide the next treatment, which is gradually being accepted and applied in clinical work.

With the advancement of biopsy equipment and technology, the current studies revealed that the firsttime biopsy diagnosis rate is about $84.6 \%-92.3 \%[4,7-12]$. In this study, the first-time biopsy diagnosis rate was $87.2 \%(95 / 109)$.In the non-diagnostic group(14 cases), we reasoned that normal tissue was punctured and the amount of tissue punctured was insufficient for pathological diagnosis. For renal tumors that cannot be diagnosed by the first-time biopsy, a second-time biopsy can be performed, and the diagnosis rate of the second-time biopsy is about $80 \%-100 \%[4,7,12,13]$. In univariate analysis, solid tumor (or: $3.208 ; 95 \% \mathrm{Ci}, 0.99-10.40, \mathrm{p}=0.052$ ) and number of biopsy cores (or: $10 ; 95 \% \mathrm{Ci}, 2.43-41.22, \mathrm{p}=$ 0.001 ) were associated with the diagnostic biopsy of this study. The multivariate analysis of no cystic lesion, number of biopsy cores, and exophytic appearance showed that solid tumor (or: $4.68 ; 95 \% \mathrm{Ci}$, $1.22-17.99, p=0.025$ ) and the number of biopsy cores (or: $9.22 ; 95 \% \mathrm{Ci}, 1.92-44.15, \mathrm{p}=0.005$ ) were significant predictors for diagnostic biopsy. Currently, a number of studies had analyzed the predictors of the diagnostic biopsy and reached different conclusions. Jeon HG's study on 393 diagnostic biopsies and 49 non-diagnostic biopsies found that small tumor size, cystic nature of tumors, and a biopsy during the initial years of the study were independent predictors of a non-diagnostic biopsy ${ }^{[7]}$. The study of Cooper $S$ et al concluded that larger tumor size (OR: $1.71 ; 95 \% \mathrm{Cl}, 1.17-2.50)$ and an exophytic location (OR: 2.91; $95 \% \mathrm{Cl}, 1.56-5.44)$ were the factors associated with a diagnostic biopsy[6]. Posielski's research suggests that cystic features of the tumor, hypo-enhancement on imaging, a smaller mass diameter and a longer skin-to-tumor distance increase the risk of non-diagnostic findings ${ }^{[14]}$.

As an invasive way of diagnosis, PRMB can obtain important information for the next treatment options such as benign or malignant lesions and histopathologic tying and grade of renal cancer. The current researches believe that the accuracy of PRMB in differentiating benign renal masses or malignant renal masses is about $97.1 \%-100 \%[7,8,12,13,15]$, and the accuracy for the diagnosis of renal tumor types is about $91 \%-98 \%[4,7,13,15,16]$, both of which have reached a high level. Nevertheless, the accuracy of grade diagnosis is not good enough, about $46 \%-69 \%[4,7,12,13,17]$. Lebret compared the pathologic results of biopsy and surgery with 85 patients and found that the concordance was $58 \%$ (49/85), while, when grades were pooled into low (G1-G2) and high (G3) - G4) grades, the concordance was much higher $(81 \%)$. In this study, 16 patients in the diagnostic group had received surgeries. With surgery pathology as the gold standard, the accuracy of PRMB in differentiating benign or malignant lesion, typing, and grading were $100 \%(16 / 16), 85.7 \%(12 / 14)$, and $50 \%(2 / 4)$, respectively.

In recent years, in addition to traditional surgical treatments (such as open and laparoscopic radical nephrectomy, partial nephrectomy, etc.), many more minimally invasive treatment options have begun to be gradually applied to the early renal tumors, such as cryotherapy, radiofrequency, and microwave ablation technology, etc. For some renal tumors with smaller diameter, it is relatively difficult to judge the benign or malignant nature only by imaging. However, if the pathological information can be obtained through PRMB, the treatment plans may be changed. In this study, 10 patients $(9.2 \%)$ had changed the 
treatment plans because the biopsy pathological results were different from the previous imaging diagnosis results (see Table 4). Some recent studies also suggest that PRMB can change a part of the treatment plans of patients with renal mass, but the treatment change rate is varied due to the different identification criteria for the treatment change group. Richard's study defined that all patients biopsied with benign renal tumors had changed their treatment plans, and the proportion of patients with treatment plans changed was $26 \%{ }^{[4]}$. The Azawi NH's study believes that the treatment plan had been changed in 35\% (45/129) patients due to benign findings or the discovery of non-renal cell cancers after biopsy ${ }^{[18]}$. Interestingly, Another study found that routine use of PRMB associated with lower rates of benign histology following nephrectomy for small renal masse ${ }^{[19]}$. For renal tumors, not only the pathological results can be obtained through PRMB, but also the tissue punctured can be used to genetic examinations, so that the pathological results and biological characteristics can be used to formulate the personalized and precise treatment plan.

Common complications of PRMB include renal hemorrhage, renal peripheral hemorrhage, postoperative pain, hematuria, pneumothorax, needle tract seeding, puncture site infection, etc. With the advancement of imaging technology and the application of automatic biopsy guns, the incidence of these complications has been much lower. Bleeding in the kidney and its peripheral tissues accounts for $75 \%$ of all complications, with an incidence of $2.1-7.6 \%$, and most of them can be cured after conservative treatment, a few need interventional therapy, however, other serious complications such as persistent hematuria and massive retroperitoneum bleeding, arteriovenous fistula are rare ${ }^{[8,14,20,21]}$.In this study, 10 patients (9.2\%) developed postoperative complications, mainly perirenal hematoma (40\%), as shown in Table 5. Serious complications (Clavien-Dindo classification,>grade 2) in the postoperative period were not observed, and no needle tract seeding was observed in the follow-ups. It is worth paying attention to needle tract seeding as a serious complication, although the incidence is extremely low, there are still sporadical cases reported ${ }^{[22-24]}$. According to our research results combined with recent similar literatures, PRMB is a relatively safe diagnostic method.

In conclusion, imaging-guided PRMB is safe with high diagnostic accuracy; it is a reasonable supplement to imaging diagnosis and may be particularly useful for inconspicuous and rare lesions for making the treatment plan.

\section{Declarations}

\section{Acknowledgements:}

This study was funded by Startup Fund For scientific research of Fujian Medical University(Grant number: 2019QH1176)

\section{Author contributions:}


Songmao Chen: Project development, Data Collection, Manuscript writing

Yuandong Chen: Manuscript writing

Liefu Ye: Project development

Qingguo Zu: Statistical analysis

Zesong Yang: Data collection

Minxiong Hu: Data collection

\section{Ethics in Publishing》}

The authors indicate this study was in accordance with the ethical standards of the World Medical Association (Declaration of Helsinki), and the study received permission from the ethics committee of Fujian Provincial Hospital(K2021-08-010). This study was a retrospective study. The data are anonymous, and the requirement for informed consent was therefore waived.

\section{Consent for publication:}

All authors have agreed to publish the current document.

\section{Disclosure区}

The authors declare no competing interests.

\section{Additional information $\rrbracket$}

Correspondence and requests for materials should be addressed to L.Y.

\section{References}

1. Capitanio U, Bensalah K, Bex A, et al. Epidemiology of Renal Cell Carcinoma. Eur Urol. 2019. 75(1): 74-84.

2. Siegel RL, Miller KD, Jemal A. Cancer statistics, 2019. CA Cancer J Clin. 2019. 69(1): 7-34.

3. Frank I, Blute ML, Cheville JC, Lohse CM, Weaver AL, Zincke H. Solid renal tumors: an analysis of pathological features related to tumor size. J Urol. 2003. 170(6 Pt 1): 2217-20.

4. Richard PO, Jewett MA, Bhatt JR, et al. Renal Tumor Biopsy for Small Renal Masses: A Single-center 13-year Experience. Eur Urol. 2015. 68(6): 1007-13. 
5. Schachter LR, Cookson MS, Chang SS, et al. Second prize: frequency of benign renal cortical tumors and histologic subtypes based on size in a contemporary series: what to tell our patients. J Endourol. 2007. 21(8): 819-23.

6. Bauman TM, Potretzke AM, Wright AJ, Knight BA, Vetter JM, Figenshau RS. Partial Nephrectomy for Presumed Renal-Cell Carcinoma: Incidence, Predictors, and Perioperative Outcomes of Benign Lesions. J Endourol. 2017. 31(4): 412-417.

7. Jeon HG, Seo SI, Jeong BC, et al. Percutaneous Kidney Biopsy for a Small Renal Mass: A Critical Appraisal of Results. J Urol. 2016. 195(3): 568-73.

8. Alle N, Tan N, Huss J, Huang J, Pantuck A, Raman SS. Percutaneous image-guided core biopsy of solid renal masses: analysis of safety, efficacy, pathologic interpretation, and clinical significance. Abdom Radiol (NY). 2018. 43(7): 1813-1819.

9. Cooper S, Flood TA, Khodary ME, et al. Diagnostic Yield and Complication Rate in Percutaneous Needle Biopsy of Renal Hilar Masses With Comparison With Renal Cortical Mass Biopsies in a Cohort of 195 Patients. AJR Am J Roentgenol. 2019. 212(3): 570-575.

10. Dave CN, Seifman B, Chennamsetty A, et al. Office-based Ultrasound-guided Renal Core Biopsy Is Safe and Efficacious in the Management of Small Renal Masses. Urology. 2017. 102: 26-30.

11. Bernhard JC, Bigot P, Pignot G, et al. The accuracy of renal tumor biopsy: analysis from a national prospective study. World J Urol. 2015. 33(8): 1205-11.

12. Richard PO, Jewett MA, Tanguay $S$, et al. Safety, reliability and accuracy of small renal tumour biopsies: results from a multi-institution registry. BJU Int. 2017. 119(4): 543-549.

13. Menogue SR, O\&\#39, Brien BA, Brown AL, Cohen RJ. Percutaneous core biopsy of small renal mass lesions: a diagnostic tool to better stratify patients for surgical intervention. BJU Int. 2013. 111(4 Pt B): E146-51.

14. Posielski NM, Bui A, Wells SA, et al. Risk Factors for Complications and Nondiagnostic Results following 1,155 Consecutive Percutaneous Core Renal Mass Biopsies. J Urol. 2019. 201(6): 10801087.

15. Wang $X, L v Y, X u Z$, et al. Accuracy and safety of ultrasound-guided percutaneous needle core biopsy of renal masses: A single center experience in China. Medicine (Baltimore). 2018. 97(13): e0178.

16. Schmidbauer J, Remzi M, Memarsadeghi M, et al. Diagnostic accuracy of computed tomographyguided percutaneous biopsy of renal masses. Eur Urol. 2008. 53(5): 1003-11.

17. Lebret T, Poulain JE, Molinie V, et al. Percutaneous core biopsy for renal masses: indications, accuracy and results. J Urol. 2007. 178(4 Pt 1): 1184-8; discussion 1188.

18. Azawi NH, Tolouee SA, Madsen M, Berg KD, Dahl C, Fode M. Core needle biopsy clarify the histology of the small renal masses and may prevent overtreatment. Int Urol Nephrol. 2018. 50(7): 1205-1209.

19. Richard PO, Lavallée LT, Pouliot F, et al. Is Routine Renal Tumor Biopsy Associated with Lower Rates of Benign Histology following Nephrectomy for Small Renal Masses. J Urol. 2018. 200(4): 731-736. 
20. Amaral BS, Macek P, Arora A, et al. Renal Tumor Biopsy: Rationale to Avoid Surgery in Small Renal Masses. Curr Urol Rep. 2021. 22(9): 46.

21. Tolouee SA, Madsen M, Berg KD, Dahl C, Fode M, Azawi NH. Renal tumor biopsies are associated with a low complication rate. Scand J Urol. 2018. 52(5-6): 407-410.

22. Soares D, Ahmadi N, Crainic O, Boulas J. Papillary Renal Cell Carcinoma Seeding along a Percutaneous Biopsy Tract. Case Rep Urol. 2015. 2015: 925254.

23. Mullins JK, Rodriguez R. Renal cell carcinoma seeding of a percutaneous biopsy tract. Can Urol Assoc J. 2013. 7(3-4): E176-9.

24. Macklin PS, Sullivan ME, Tapping CR, et al. Tumour Seeding in the Tract of Percutaneous Renal Tumour Biopsy: A Report on Seven Cases from a UK Tertiary Referral Centre. Eur Urol. 2019. 75(5): 861-867. 